\title{
An update of aberrant methylation detection on circulating cell-free DNA as a tool to improve prostate cancer diagnosis and prognosis
}

\author{
Nicola Pavan', Gabriele Grassi ${ }^{2}$, Bruna Scaggiante ${ }^{2}$ \\ 'Department of Medical, Surgical and Health Science, Urology Clinic, University of Trieste, Trieste 34127, Italy. \\ ${ }^{2}$ Department of Life Sciences, University of Trieste, Trieste 34127, Italy.
}

Correspondence to: Prof. Bruna Scaggiante, Department of Life Sciences, University of Trieste, via Valerio 28/1, Trieste 34127, Italy. E-mail: bscaggiante@units.it

How to cite this article: Pavan N, Grassi G, Scaggiante B. An update of aberrant methylation detection on circulating cell-free DNA as a tool to improve prostate cancer diagnosis and prognosis. J Trans/ Genet Genom 2021;5:173-81.

https://dx.doi.org/10.20517/jtgg.2021.18

Received: 31 Mar 2021 Accepted: 28 May 2021 First online: 2 Jun 2021

Academic Editor: Sanjay Gupta Copy Editor: Xi-Jun Chen Production Editor: Xi-Jun Chen

\begin{abstract}
Prostate cancer ( $\mathrm{PCa}$ ) remains a disease of high incidence, but orphan of a specific screening program. For this reason, non-invasive techniques capable to predict $\mathrm{PCa}$ in patients with high specificity and sensitivity are still an urgent need. One of the major goals is to improve the PCa diagnosis and the identification of patients who benefit from tissue biopsies. Another need is the necessity to have novel biomarkers to better stratify the risk of patients with PCa to predict the aggressiveness of the tumor and the overall survival. Liquid biopsy can be an important non-invasive tool to stratify PCa at the molecular level to improve diagnosis and prognosis, and, possibly, to develop screening programs and follow-up. With this review, we are reporting the lastest update of aberrant methylation detection on circulating tumor DNA as a tool to improve prostate cancer diagnosis and prognosis.
\end{abstract}

Keywords: Liquid biopsy, DNA, methylation, prostate cancer, cfDNA, ctDNA, biomarker

\section{INTRODUCTION}

Prostate cancer (PCa) is the main cause of cancer-related death in men in Western countries ${ }^{[1]}$. Screening programs to identify adenocarcinoma by the prostate-specific antigen (PSA) have a specificity too low to be 
largely proposed as the first-line test. Moreover, PSA is elevated as well in benign prostate pathologies, and about $15 \%$ of asymptomatic PCa patients do not present elevated PSA levels. Nowadays, prostate biopsy remains the procedure necessary for the diagnosis of PCa despite the invasiveness, possible side effects and cost of the procedure.

More specific PCa biomarkers are urgently needed not only to anticipate cancer diagnosis, especially the identification of the more aggressive forms, but also for the best management of therapeutic interventions and the surveillance of cancer progression. In particular, for patients in active surveillance (AS), a more specific test and less invasive procedure are needed to improve the adherence to the protocols and maintain a good quality of life for patients.

At the genomic level, tumor onset and progression are importantly modulated at the epigenetic level by the DNA methylation changes in specific regions, mainly at gene promoter sites. The epigenetic modulation of DNA is a field of intense research to find novel biomarkers for diagnosis/prognosis or targets for innovative therapeutic strategies. Methylation of the $\mathrm{CpG}$ islands is a very frequent aberration in cancer that occurs also in $\mathrm{PCa}^{[2]}$. Thus, a part of the scientific interest is directed to the "methylome" analysis of the DNA to better define the onset and the phenotype evolution of cancer.

To analyze tumor DNA, liquid biopsy can offer a non-invasive tool to monitor specific PCa biomarkers in different biological fluids. Nowadays, it is possible to detect, with high sensitivity and specificity, circulating tumor nucleic acids (DNA and RNA) derived from cancer cells that have died. For example, the search of DNA aberrations of the androgen receptor gene has been found useful at a prognostic and predictive level and they are strongly correlated with patients' outcomes ${ }^{[3]}$.

This mini-review summarizes the major and recent discoveries of aberration in the methylation pattern of circulating tumor DNA. We mainly focused on the biomarkers that have been demonstrated to be clinically useful or promising.

A literature review was performed using PubMed and three main key terms (DNA methylation AND prostate cancer AND circulating cell-free DNA AND liquid biopsy) We selected research articles from 2015 to 2021. Articles included in this review are summarized in Table 1.

\section{METHYLATED BIOMARKERS IN CIRCULATING CELL-FREE DNA FROM URINE} The cfDNA containing ctDNA has been collected after digital rectal examen or at first void

One of the most interesting translational research comes from Brikun et al. ${ }^{[4,5]}$. Using cell-free DNA (cfDNA) from urine after digital rectal examen (DRE) or at first void (FV), they demonstrated, using a panel of 19 targets that the number of methylated markers was statistically higher in PCa cases compared to controls, 10 of 19 vs. 3 of 19, respectively. Six of nineteen methylated markers (6 of 19) were shown to be the threshold to predict PCa with a negative predictive value (NPV) $\geq 90 \%$ for both DRE and FV urine cfDNA. In addition, authors proved a significative association between the number of methylated markers and the PCa diagnosis with the tissue biopsies. Finally, in post-DRE urine samples, a higher rate of biomarkers was reported when compared to urine at FV. In particular, AOX1, coding an aldehyde oxidase that regulates reactive oxygen species homeostasis, GRFA2, coding a neurotrophic factor involved in cell survival and differentiation, and NEUROG3, coding a transcriptional regulator, cannot be found in FV samples ${ }^{[5]}$.

Furthermore, in a subsequent study the authors proved that other 13 markers can be used to predict early $\mathrm{PCa}$ or to stratify the disease. Using the same sample of cfDNA from urine after DRE or at FV, a panel of 32 
Table 1. Summary of studies

\begin{tabular}{|c|c|c|c|c|c|c|c|c|c|c|c|c|}
\hline Ref. & $\begin{array}{l}\text { Year of } \\
\text { publication }\end{array}$ & $\begin{array}{l}\text { Number } \\
\text { of } \\
\text { patients }\end{array}$ & Cases & $\begin{array}{l}\text { Control } \\
\text { group }\end{array}$ & Sample & Methodology & Biomarker & $\begin{array}{l}\text { Diagnostic } \\
\text { value }\end{array}$ & $\begin{array}{l}\text { Prognostic } \\
\text { value }\end{array}$ & $\begin{array}{l}\text { Tissue } \\
\text { concordance }\end{array}$ & Specificity & Sensitivity \\
\hline Reis et al. ${ }^{[11]}$ & 2015 & 82 & $34 \mathrm{PCa}$ & $\begin{array}{l}48 \\
\text { healthy } \\
\text { control }\end{array}$ & Serum & $\begin{array}{l}\text { Ms-SNuPE and } \\
\text { pyrosequencing }\end{array}$ & GADD45a & Yes & Yes & No & $87 \%$ & $94 \%$ \\
\hline Haldrup et al. ${ }^{[10]}$ & 2018 & 815 & $\begin{array}{l}705 \\
\text { PCa/ZNF660 } \\
\text { tested in } \\
\text { different } \\
\text { cohorts }\end{array}$ & $\begin{array}{l}110 \\
\text { healthy } \\
\text { control }\end{array}$ & Serum & MS-qPCR & $\begin{array}{l}\text { ST6GALNAC3, } \\
\text { ZNF660, HAPLN3 }\end{array}$ & Yes & Yes & Yes & $100 \%$ & $67 \%$ \\
\hline Menschikowski et al. ${ }^{[13]}$ & 2018 & 2 & $2 \mathrm{PCa}$ & NA & Serum & OBBPA-ddPCR & PLA2R1 & Yes & No & No & NA & NA \\
\hline Brikun et al. $^{[5]}$ & 2018 & 94 & $42 \mathrm{PCa}$ & $\begin{array}{l}52 \\
\text { healthy } \\
\text { control }\end{array}$ & $\begin{array}{l}\text { Urine (after } \\
\text { DRE and at } \\
\text { FV) }\end{array}$ & $\begin{array}{l}\text { MS-qPCR by binary } \\
\text { presence }(>0) \text { or } \\
\text { absence }(<0) \text { of } \\
\text { methylation }\end{array}$ & $\begin{array}{l}\text { ADCY4, AOX1rc, } \\
\text { APC, CXCL14, } \\
\text { EPHX3, GFRA2, } \\
\text { GSTP1, HEMK1rc, } \\
\text { KIFC2, MOXD1, } \\
\text { HOXA7, HOXB5, } \\
\text { HOXD3, HOXD9, } \\
\text { HOXD10, HOXD3a, } \\
\text { HOXD3b, } \\
\text { NEUROG3, } \\
\text { NODAL, RASSF5 }\end{array}$ & Yes & No & No & $\begin{array}{l}71 \% \text { after } \\
\text { DRE and FV }\end{array}$ & $\begin{array}{l}89 \% \text { after } \\
\text { DRE and } \\
94 \% \text { from } \\
\text { FV }\end{array}$ \\
\hline Brikun et al. ${ }^{[4]}$ & 2019 & 94 & $42 \mathrm{PCa}$ & $\begin{array}{l}52 \\
\text { healthy } \\
\text { control }\end{array}$ & $\begin{array}{l}\text { Urine ( } 85 \\
\text { after DRE and } \\
65 \text { at FV) }\end{array}$ & $\begin{array}{l}\text { MS-qPCR by binary } \\
\text { presence }(>0) \text { or } \\
\text { absence }(<0) \text { of } \\
\text { methylation }\end{array}$ & $\begin{array}{l}\text { PANEL ABOVE } \\
\text { PLUS 13 additional } \\
\text { markers: } \\
\text { HOXA11as, KLK10, } \\
\text { GPR147, GPR62, } \\
\text { HOXD4rc, } \\
\text { HOXD3c, FRZB, } \\
\text { GRASPrc, } \\
\text { HOXBAS3, } \\
\text { HOXD8rc, RASSF1, } \\
\text { SLC16A5rc }\end{array}$ & Yes & No & No & $\begin{array}{l}76 \% \text { from } \\
\text { DRE- } 77 \% \\
\text { after FV }\end{array}$ & $\begin{array}{l}81 \% \text { from } \\
\text { DRE- } 93 \% \\
\text { after FV }\end{array}$ \\
\hline Nekrasov et al. ${ }^{[6]}$ & 2019 & 64 & $31 \mathrm{PCa}$ & $\begin{array}{l}33 \\
\text { healthy } \\
\text { control }\end{array}$ & Urine & qMSP & $\begin{array}{l}\text { APC2, CDH1, } \\
\text { FOXP1, FOXP2, } \\
\text { FOXP3, FOXP4, } \\
\text { H1C1, HOXA9, } \\
\text { LRRC3B, MGMT, } \\
\text { NDRG4, PLCL2, } \\
\text { PTEN, UBE2E2, } \\
\text { VHL, WNT7A, } \\
\text { ZIC4 }\end{array}$ & Yes & Yes & No & $100 \%$ & $78 \%$ \\
\hline O'Reilly et al. ${ }^{[16]}$ & 2019 & 463 & $209 \mathrm{PCa} / 31+$ & 254 & Urine & HumanMethylation 450 & Methylation array & Yes & Yes & Yes & $76 \%$ & $73 \%$ \\
\hline
\end{tabular}




\begin{tabular}{|c|c|c|c|c|c|c|c|c|c|c|c|c|}
\hline & & & $\begin{array}{l}178 \text { tissue } \\
\text { samples }\end{array}$ & $\begin{array}{l}\text { healthy } \\
\text { control }\end{array}$ & & BeadChip (HM450k) & chip & & & & & \\
\hline Connell et al. ${ }^{[7]}$ & 2020 & 207 & $207 \mathrm{PCa}$ & NA & Urine & MS-qPCR & $\begin{array}{l}\text { GSTP1, SRFP2, } \\
\text { IGFBP3, IGFBP7, } \\
\text { APC, PTSG2 }\end{array}$ & Yes & Yes & No & NA & NA \\
\hline Zhao et al. ${ }^{[8]}$ & 2019 & 103 & 103 PCa on AS & NA & Urine & $\begin{array}{l}\text { Multiplex MethyLight } \\
\text { assay }\end{array}$ & $\begin{array}{l}\text { APC, GSTP1, CRIP3, } \\
\text { HOXD8 }\end{array}$ & No & Yes & No & $60 \%$ & $81 \%$ \\
\hline Constâncio et al. ${ }^{[12]}$ & 2019 & 121 & $121 \mathrm{PCa}$ & NA & Plasma & Multiplex qMSP & $\begin{array}{l}\text { APC, FOXA1, } \\
\text { GSTP1, HOXD3, } \\
\text { RAR } 32, \text { RASSF1A, } \\
\text { SEPT9, SOX17 }\end{array}$ & Yes & Yes & No & $72 \%$ & $72 \%$ \\
\hline Carson et al. ${ }^{[17]}$ & 2020 & ND & $\begin{array}{l}\text { Small cohort } \\
\text { of patients of } \\
\text { men with BCR }\end{array}$ & NA & Serum/Plama & mDETECT & $\begin{array}{l}\text { Methylation } \\
\text { DETection of } \\
\text { circulating tumor } \\
\text { DNA }\end{array}$ & No & Yes & No & NA & NA \\
\hline Bjerre et al. ${ }^{[14]}$ & 2020 & 264 & $\begin{array}{l}102 \mathrm{IPCa}, 65 \\
\text { de novo mPCa }\end{array}$ & $\begin{array}{l}36 \\
\text { healthy } \\
\text { control, } \\
61 \mathrm{BPH}\end{array}$ & Plasma & MS-ddPCR & $\begin{array}{l}\text { DOCK2, HAPLN3, } \\
\text { FBXO3O }\end{array}$ & Yes & Yes & Yes & $80 \%-100 \%$ & $75 \%-94 \%$ \\
\hline Beltran et al. ${ }^{[15]}$ & 2020 & 62 & $\begin{array}{l}10 \mathrm{mPCa}, 35 \\
\text { CRPC-adeno, } \\
17 \text { CRPC-NE }\end{array}$ & NA & Plasma & WGBS & $\begin{array}{l}\text { Relevant from panel } \\
\text { of genes: ASXL3, } \\
\text { SPDEF, INSM1, } \\
\text { CDH2, TP53, RB1, } \\
\text { CYLD, AR }\end{array}$ & Yes & No & Yes & NA & NA \\
\hline Wu et al. ${ }^{[18]}$ & 2020 & 25 & $25 \mathrm{mCRP}$ & NA & Plasma & NGS & $\begin{array}{l}\text { Next-generation } \\
\text { sequencing on } \\
\text { plasma DNA }\end{array}$ & Yes & No & No & NA & NA \\
\hline Silva et al. ${ }^{[9]}$ & 2020 & 4 & $4 \mathrm{mCRP}$ & NA & $\begin{array}{l}\text { Urine and } \\
\text { plasma }\end{array}$ & $\begin{array}{l}\text { MethyLight qPCR and } \\
\text { Infinium }{ }^{\circledR} \\
\text { MethylationEPIC } \\
\text { BeadChip }\end{array}$ & $\begin{array}{l}\text { Methylation array } \\
\text { chip }\end{array}$ & Yes & No & Yes & NA & NA \\
\hline
\end{tabular}

AS: Active surveillance; BCR: biochemical recurrence; BPH: benign prostatic hyperplasia; CRPC-adeno: castration-resistant adenocarcinoma; ddPCR: digital droplet PCR; DRE: digital rectal examination; FV: first morning void; IPCa: localized prostate cancer; qPCR: quantitative PCR; qMPS: quantitative Methylation specific PCR; mCRPC-NE: metastatic castration-resistant neuroendocrine prostate cancer; mCRP: metastatic castration resistant prostate cancer; mDETECT: methylation DETection of circulating tumor DNA; mPCa: hormone-naive metastatic prostate adenocarcinoma; PCa: prostate cancer; MS-SNuPE: Microarray-based methylation-sensitive single-nucleotide primer extension; MS-ddPCR: methylation specific digital droplet PCR: MS-qPCR: methylation specific quantitative PCR: Multiplex qMSP: multiplex quantitative methylationspecific PCR; NA: not assested; ND: not defined; NGS: next-generation sequencing; OBBPA-ddPCR: optimized bias-based pre-amplification-digital droplet PCR; WGBS: whole-genome bisulfite sequencing.

markers was proposed and tested ${ }^{[4]}$. They found that in both groups, the median number of the methylated markers was higher in PCa cases than controls, 16 of $32 \mathrm{vs} .5$ of 32, respectively. The 10 of 32 positive methylated markers cutoff was found to be the threshold to recommend a patient for prostate biopsy. The positive predictive value did not significantly improve from the previous study with 19 targets, being $71 \%$ and $77 \%$, for DRE and FV, respectively. On the 
contrary, the NPV was confirmed to have high performance being $85 \%$ and $93 \%$, for DRE and FV, respectively. Both studies showed a significant increase in the area under the curve (AUC) values of the Receiver Operating Characteristic (ROC) curves when compared to PSA. This finding demonstrates the higher specificity and sensitivity of the number of methylated markers in urinary cfDNA compared to PSA level. Notably, HOXD3 and HOXA7, both encoding members of the family of transcription factors, GPR62, coding a signaling factor of the phosphoinositol pathway, and KLK10, coding a serine protease implicated in carcinogenesis, were found in all PCa samples; however, HOXD8rc, encoding a member of the family of transcription factors, CXCL14, encoding a protein involved in inflammatory and immunomodulatory functions, SLC16A5rc, encoding a member of a family of carrier, and GRASP, encoding a scaffold protein involved in phosphoinositide pathway, were more frequently present in highly aggressive PCa, thus suggesting for the last ones a prognostic value for these markers.

Moreover, both studies evaluated the correlation between the number of methylated markers or the average of methylation with the risk score University of California San Francisco Cancer of the Prostate Risk Assessment. Overall, the results suggested a high performance of the methylation test to identify patients at risk of $\mathrm{PCa}$ and the possibility to use these markers and their global status of methylation to stratify patients for PCa aggressiveness. The 32-panel of biomarkers has improved the precision for patient stratification by giving the indication for biopsy in those patients who did not reach the threshold in the 19-panel. Notably, the possibility to determine the risk stratification was assigned to urine CfDNA after DRE, because of the possibility to recover more cancer cells and to avoid dilution and degradation of DNA derived from urine at FV specimens that may cause a higher sampling error. However, the authors underline that in 58 patients, both DRE and FV samples were equivalent in the analysis results, thus suggesting that FV remains a useful and simple source for cfDNA. These markers matched with the age of patients and others anamnestic parameters could improve the sensitivity/specificity of the test. A future dedicated clinical trial will be able to find the clinical correlations necessary for the validations of these markers.

Nekrasov et al. ${ }^{[6]}$ collected 31 urine samples from PCa patients and 33 samples in healthy patients as diseasefree control. The methylation status of 17 cancer-associated genes was analyzed using a methylation-specific polymerase chain reaction. They reported 13 genes with increased methylation frequency in patients with PCa compared with the control group. In conclusion, the authors reported a 6-gene panel (APC2, CDH1, FOXP1, LRRC3B, WNT7A, and ZIC4) able to identify PCa with $78 \%$ sensitivity and 100\% specificity.

Connel et al..$^{[7]}$, reported a multivariable risk model integrating urinary cell DNA methylation and cfRNA data able to detect significant PCa. In their analysis, 207 post-digital rectal examination urine samples were collected within a Movember cohort (GAP1 urine biomarker). ExoMeth was the name of the model created for this study. Clinical variables (age and PSA) were integrated with methylation and transcript targets. The model was subsequently tested and applied to a final cohort of 197 with available data. With an odds ratio (OR) of 2.04 (95\%CI: 1.78-2.35) per 0.1 ExoMeth increase, they were able to increase the likelihood highgrade of the disease being detected on prostate biopsy. In the future, this can potentially avoid unnecessary biopsies in patients on AS or to guide the necessity of mpMRI in patients with a clinically suspected PCa.

Similarly, Zhao et al. ${ }^{[8]}$ combined the urinary DNA methylation with cf-mRNA biomarkers in a series of 103 $\mathrm{CaP}$ patients on AS. The aim of the study was the identification of patients at risk of reclassification. Three marker panels (miR-24, miR-30c and CRIP3 methylation) were identified in the post-DRE urinary sediment using a qPCR-based MethyLight assay. With a NPV of 90\% and an OR of 2.17 (95\%CI: 1.22-3.85), the authors were able to identify patients with a PCa progression. CRIP3 methylation was found to be a significant predictor of AS reclassification ( $\mathrm{OR}=1.079,95 \% \mathrm{CI}$ : 1.013-1.15). 
Silva et al. ${ }^{[9]}$, designed a prospective study to investigate the role of blood and urine in capturing the PCa methylome. They selected a cohort of 4 patients with de novo metastatic $\mathrm{PCa}(\mathrm{mPCa})$ and a post-DRE and FV sample of urine were analyzed. Detection of tumor DNA methylation probes in urine ranged from 6.98\% to $39.40 \%$. Authors demonstrated, through a DNA methylation analysis, highly correlated patterns between the different liquid types ( $\rho=0.93, P<0.0001$ ), with large contributions from non-tumor sources.

Finally, a promising Danish study ${ }^{[10]}$ investigated the role of novel aberrant promoter hypermethylation of specific genes to improve the diagnosis and prognosis of PCa. Methylation in the promoter region of genes was analyzed in prostate tissue and liquid biopsy. In particular, ST6GALNAC3 encoding a member of the sialyltransferases for the modification of glycoproteins, ZNF660 encoding a transcriptional regulator, T6GALNAC3 encoding a protein identified in neutrophil granules, ZNF660 encoding a transcription factor, CCDC181 encoding a microtubule binding protein, and HAPLN3 encoding a membrane protein. 815 samples (705 PCa and 110 non-cancer) were processed by methylation-specific qPCR or methylation array. The AUC of the ROC analysis demonstrated the role of hypermethylation of ST6GALNAC3 and ZNF660 in the diagnosis of PCa (0.917-0.995 vs. 0.846-0.903 in cancer $v s$. non-cancer samples, respectively). Moreover, ZNF660 hypermethylation was tested in two radical prostatectomy cohorts of 158 and 392 patients.

ZNF660 hypermethylation was also significantly associated with poor overall and PCa-specific survival in a different cohort of radical prostatectomy $(n=158)$ with long clinical follow-up available showing a potential prognostic role. In the same study, a panel of hypermethylated circulating tumor DNA (ctDNA) for ST6GALNAC3, ZNF660, HAPLN3, and CCDC181 was proposed for liquid biopsy. A final ctDNA hypermethylation model of 3 genes (ST6GAL-NAC3/CCDC181/HAPLN3) was developed with 100\% of specificity and $67 \%$ of sensitivity in the detection of PCa.

\section{METHYLATED BIOMARKERS IN CIRCULATING CELL-FREE DNA FROM BLOOD} The cfDNA, containing ctDNA, has been collected from plasma or from serum

In 2015, Reis et al. ${ }^{[11]}$ studied, in serum, cfDNA the methylation of GADD45a gene, which they previously found to be methylated at different sites in tissue PCa tissue. The authors found a statistically significant difference between the methylation of GADD45a in Pca with respect to Benign Prostatic Hyperplasia (BPH) patients' serum. The PCa samples were more methylated than BPH controls, although in PCa patients a higher methylation variability than $\mathrm{BPH}$ controls was found. No correlation between GADD45a methylation and Gleason score was evidenced. Interestingly, the methylation status of GADD45a and the PSA level better define PCa versus BPH patients than GADD45a methylation alone.

The role of aberrant DNA promoter methylation was also studied as a possible tool for simultaneous detection of several types of cancers ${ }^{[12]}$. In a large multicenter study, this hypothesis was tested for lung, prostate, and colorectal cancers. More deeply, cfDNA was extracted from 121 PCa patients and the level of methylation of different promoters was assessed. The authors proposed a "Pan-Cancer" panel (FOXA1me, RARß2me and RASSF1Ame) able to simultaneously detect PCa and lung cancer (SP 70\% and SS 64\%). The panel was also able to discriminate between intermediate and high-risk PCa with a sensitivity of $71 \%$ and a specificity of $65 \%$. These results can be interesting when future studies will apply this panel in an AS setting or in the decision-making process for a diagnostic biopsy in the suspected cases of PCa.

As previously described, the study published by Silva et al..$^{[9]}$ analyzed the role of blood in capturing DNA methylation. Utilizing the Infinium ${ }^{\circ}$ MethylationEPIC BeadChip (Illumina) they were able to detect DNA methylation probes from $7.19 \%$ to $64.14 \%$ in plasma. Matching liquid and prostate biopsies controls authors prevented the effect of unwanted variables and reduced the inter-individual variability. Despite the small 
number of patients, the authors have shown that both plasma and urine serve as excellent surrogates for detecting tumoral epigenomic alterations.

Menschikowski et al ${ }^{[13]}$ developed a novel amplification system based on digital-droplet PCR (ddPCR), named optimized bias-based pre-amplification ddPCR (OBBPA-ddPCR), for early detection of rare DNA methylation targets. They demonstrated that this technique can specifically detect PLA2R1 gene methylation in serum of PCa patients with a very high sensitivity. PLA2R1 encodes a phospholipase A2 receptor. If this novel assay could be usable for the early identification of PCa patients remains to be demonstrated in a large sample cohort.

Bjerre et al. ${ }^{[14]}$ proved from a panel of 24 candidate biomarkers that three of them, DOCKK2, HAPLN3, encoding an important protein that binds hyaluronic acid involved in many cellular function and cell adhesion, and FBXO30, encoding a member of F-box protein family involved in protein degradation, were strongly related to the progression of hormone-naïve mPCa to castration-resistant mPCa. They used plasma samples that were analyzed by MS-ddPCR. Interestingly, these markers did not result in methylation in healthy controls, $\mathrm{BPH}$, or localized PCa patients. The authors noted that plasma cfDNA quantity did not differ between healthy donors, $\mathrm{BPH}$, localized PCa, or de novo mPCa patients. However, a higher level of cfDNA was found to be related to cases in a more advanced clinical stage. It is important to underline that in $\mathrm{BPH}$ or localized PCa samples, the cfDNA methylation in the biomarkers was rarely found; on the contrary, in corresponding tissue samples, the methylation of all markers was present. The markers were highly sensitive and specific to identify high tumor volume, de novo mPCa. From the clinical point of view, the methylation of any of the three biomarkers was related with shorter OS in these patients, as an independent predictor.

Beltran et al. ${ }^{[15]}$ studied cfDNA in castration-resistant neuroendocrine prostate cancer (CRPC-NE). A significant proportion of $\mathrm{PCa}$ with this phenotype are linked with a poor prognosis. They performed wholeexome sequencing in cfDNA from plasma to identify any aberration in the expression of important tumor suppressor genes such as TP53 and RB1. The same analysis was applied also in genes involved in DNA repair such as BRCA1, BRCA2, FANCA, or an important checkpoint signaling regulators such as Ataxiatelangiectasia mutated gene (ATM) gene. The authors also performed whole-exome genome bisulfite sequencing in a small sample of patients harboring CRPC-adeno or CRPC-NE and compared the results with the methylation pattern in the tissues. A concordance of the methylation status of the targets between cfDNA and tissue biopsy was found. In CRPC-NE samples hypo or hypermethylation status of 20 different sites marked this tumor phenotype in cfDNA.

\section{CONCLUSION}

The ctDNA can be easily identified from the quote of cfDNA release from cancer cells in bloodstream or in urine. It is important to note that the total level of cfDNA in plasma did not relate with presence of PCA, although higher values of cfDNA have been found in advanced disease patients ${ }^{[14]}$. However, the measure of cfDNA level alone remains a poor predictor of the disease. Methylation of specific biomarkers and, in particular, the number of methylated biomarkers in a panel can provide useful tools for clinicians either to manage the risk of asymptomatic patients or to predict the progression.

Molecular tests are more expensive than PSA, however the reduction of improper prostate biopsies, the precise identification of patients with risk should fully balance the initial screening test costs, and prospectively save costs and suffering. Reducing the number of unnecessary biopsies will be one of the best targets that we can achieve in PCa. The finding of low-grade PCa and an increase demand of AS need to be 
supported by reliable and precise test able to define an upgrading or upstaging of PCa. The reduction of costs associated to unnecessary treatment will be devolved for more precise screening and diagnosis able to improve the quality of life of our patients.

Some of the biomarkers are candidates for identifying more aggressive forms such as HOXD8rc, CXCL14, SLC16A5rc and GRASP or to predict progression such as DOCKK2, HAPLN3 and FBXO30. It is important to note that methylation of some biomarkers that can be found in tissues, cannot be found in ctDNA ${ }^{[14]}$. This is because PCa does not release cancer cells if it is at the early stages. Thus, the analysis of cfDNA methylation associated to the study of PCa tissues, remains a very important task to deepen the role of cfDNA biomarkers methylation.

In conclusion the studies showed the possibility to analyze the methylation of cfDNA biomarkers either from plasma or urine, thus opening more possibilities to monitor PCa patients and, possibly to develop screening programs. A challenge is to study the integration of the anamnestic data, the PSA and the molecular methylation status on cfDNA to personalize the patient's care.

\section{DECLARATIONS}

\section{Authors' contributions}

Made substantial contributions to conception, data search, design and writing of the review: Pavan N, Scaggiante B

Performed critical revision: Grassi G

\section{Availability of data and materials}

Not applicable.

\section{Financial support and sponsorship}

None.

\section{Conflicts of interest}

All authors declared that there are no conflicts of interest.

\section{Ethical approval and consent to participate}

Not applicable.

\section{Consent for publication}

Not applicable.

\section{Copyright}

(c) The Author(s) 2021.

\section{REFERENCES}

1. Siegel RL, Miller KD, Jemal A. Cancer statistics, 2019. CA Cancer J Clin 2018;69:7-34. DOI PubMed

2. Goering W, Kloth M, Schulz WA. DNA methylation changes in prostate cancer. In: Dumitrescu RG, Verma M, editors. Cancer epigenetics. Totowa: Humana Press; 2012. p. 47-66.

3. Sobhani N, Sirico M, Generali D, Zanconati F, Scaggiante B. Circulating cell-free nucleic acids as prognostic and therapy predictive tools for metastatic castrate-resistant prostate cancer. World J Clin Oncol 2020;11:450-63. DOI PubMed PMC

4. Brikun I, Nusskern D, Freije D. An expanded biomarker panel for the detection of prostate cancer from urine DNA. Exp Hematol Oncol 2019;8:13. DOI PubMed PMC

5. Brikun I, Nusskern D, Decatus A, Harvey E, Li L, Freije D. A panel of DNA methylation markers for the detection of prostate cancer from FV and DRE urine DNA. Clin Epigenetics 2018;10:91. DOI PubMed PMC

6. Nekrasov KA, Vikarchuk MV, Rudenko EE, et al. 6-gene promoter methylation assay is potentially applicable for prostate cancer 
clinical staging based on urine collection following prostatic massage. Oncol Lett 2019;18:6917-25. DOI PubMed PMC

7. Connell SP, O'Reilly E, Tuzova A, et al. Development of a multivariable risk model integrating urinary cell DNA methylation and cellfree RNA data for the detection of significant prostate cancer. Prostate 2020;80:547-58. DOI PubMed PMC

8. Zhao F, Vesprini D, Liu RSC, et al. Combining urinary DNA methylation and cell-free microRNA biomarkers for improved monitoring of prostate cancer patients on active surveillance. Urol Oncol 2019;37:297.e9-297.e17. DOI PubMed

9. Silva R, Moran B, Russell NM, et al. Evaluating liquid biopsies for methylomic profiling of prostate cancer. Epigenetics 2020;15:71527. DOI PubMed PMC

10. Haldrup C, Pedersen AL, Øgaard N, et al. Biomarker potential of ST6GALNAC3 and ZNF660 promoter hypermethylation in prostate cancer tissue and liquid biopsies. Mol Oncol 2018;12:545-60. DOI PubMed PMC

11. Reis IM, Ramachandran K, Speer C, Gordian E, Singal R. Serum GADD45a methylation is a useful biomarker to distinguish benign vs malignant prostate disease. Br J Cancer 2015;113:460-8. DOI PubMed PMC

12. Constâncio V, Nunes SP, Moreira-Barbosa C, et al. Early detection of the major male cancer types in blood-based liquid biopsies using a DNA methylation panel. Clin Epigenetics 2019;11:175. DOI PubMed PMC

13. Menschikowski M, Jandeck C, Friedemann M, et al. Identification of rare levels of methylated tumor DNA fragments using an optimized bias based pre-amplification-digital droplet PCR (OBBPA-ddPCR). Oncotarget 2018;9:36137-50. DOI PubMed PMC

14. Bjerre MT, Nørgaard M, Larsen OH, et al. Epigenetic analysis of circulating tumor DNA in localized and metastatic prostate cancer: evaluation of clinical biomarker potential. Cells 2020;9:1362. DOI PubMed PMC

15. Beltran H, Romanel A, Conteduca V, et al. Circulating tumor DNA profile recognizes transformation to castration-resistant neuroendocrine prostate cancer. J Clin Invest 2020;130:1653-68. DOI PubMed PMC

16. O'Reilly E, Tuzova AV, Walsh AL, et al. epiCaPture: a urine DNA methylation test for early detection of aggressive prostate cancer. JCO Precis Oncol 2019;2019:PO.18.00134. DOI PubMed PMC

17. Carson JJK, Di Lena MA, Berman DM, Siemens DR, Mueller CR. Development and initial clinical correlation of a DNA methylationbased blood test for prostate cancer. Prostate 2020;80:1038-42. DOI PubMed

18. Wu A, Cremaschi P, Wetterskog D, et al. Genome-wide plasma DNA methylation features of metastatic prostate cancer. J Clin Invest 2020;130:1991-2000. DOI PubMed PMC 